\title{
Self-consistent quantum effects in the quark meson coupling model
}

\author{
P. K. Panda ${ }^{1, *}$ and F. L. Braghin ${ }^{2, \dagger}$ \\ ${ }^{1}$ Instituto de Física Teórica, Universidade Estadual Paulista, Rua Pamplona 145, CEP 01405-900 São Paulo, SP, Brazil \\ ${ }^{2}$ Instituto de Física, Universidade de São Paulo, Caixa Postal 66318, CEP 05315-970 São Paulo, Brazil
}

(Received 10 July 2002; published 8 November 2002)

\begin{abstract}
We derive the equation of state of nuclear matter for the quark-meson coupling model taking into account quantum fluctuations of the $\sigma$ meson as well as vacuum polarization effects for the nucleons. This model incorporates explicitly quark degrees of freedom with quarks coupled to the scalar and vector mesons. Quantum fluctuations lead to a softer equation of state for nuclear matter giving a lower value of incompressibility than would be reached without quantum effects. The in-medium nucleon and $\sigma$-meson masses are also calculated in a self-consistent manner. The spectral function of the $\sigma$ meson is calculated and the $\sigma$ mass has the value increased with respect to the purely classical approximation at high densities.
\end{abstract}

DOI: 10.1103/PhysRevC.66.055207

PACS number(s): 12.39.Ba, 21.65.+f, 24.85.+p

\section{INTRODUCTION}

The study of the high density and high temperature hadronic matter is one of the most interesting subjects in nuclear many-body physics for the understanding of superdense stars and relativistic heavy-ion collision. Usually the frame of quantum hadrodynamics (QHD) [1] is the departure to the study of the nuclear many-body problem describing nucleons interacting with scalar and vector mesons. This meson field theory has quite successfully described the properties of the nuclear matter and finite nuclei using the mean field approximations for the meson fields. The vacuum polarization corrections arising from the nucleon fields as well as the meson fields have also been considered to study the nuclear matter [2]. This is one way of obtaining a softer equation of state yielding a lower compressibility than would be reached without quantum effects. This is an indication that even if one is interested in developing effective models for the description of such complex systems a description taking into account quantum fluctuations may be of relevance.

While descriptions of the nuclear phenomena have been efficiently formulated using some hadronic degrees of freedom as in QHD, there have been interesting observations that reveal the medium modification of the internal structure of the nucleon. High density and temperature matter has been, and is being, investigated in RHIC and CERN and in medium modifications of the parameters of the theory used for the description of the experimental observations must be considered. Furthermore, quantum chromodynamics (QCD) is expected to present a phase in which quarks and gluons are not confined inside hadrons at high densities and/or temperatures. At such high densities and/or temperatures, asymptotic freedom implies smaller coupling constants. Therefore the degrees of freedom from the fundamental theory of strong interacting systems, QCD, must be considered. Due to the complex structure of this theory we are led to formulate effective models that have the main properties and symmetries of QCD as chiral symmetry and its spontaneously symmetry

\footnotetext{
*Email address: panda@ift.unesp.br

${ }^{\dagger}$ Email address: braghin@if.usp.br
}

breaking. One of the first models put forward along these lines was the quark-meson coupling (QMC) $[3,4]$ model which describes the different phases of hadronic matter in terms of explicit quark degrees of freedom. This model describes nuclear matter with nucleons as nonoverlapping MIT bags interacting through the scalar and the vector mesons, very much in the same way as in the QHD [1]. The crucial difference is that in the QMC, the mesons couple directly to the quarks in the interior of the baryon. Many applications and extensions of the model have been made in the last years-see Refs. [5-10], and references therein. Among several aspects the density dependent bag constant has also been investigated within this frame [10]. The behavior of the order parameter of the chiral symmetry breaking has been focus of attention and is believed to have strong consequences for observations in relativistic heavy-ions collisions. There are evidences for believing that the symmetry is restored at high densities (and temperatures) making the order parameter to vanish although the idea of further symmetry breaking with increasing densities [11] has not been extensively studied. Although effective models are usually developed for classical mean fields, one is often driven to the investigation of the contribution of quantum effects which modify dynamical equations and observables.

In the present work, we investigate the role of the quantum fluctuations of the $\sigma$ meson in a nonperturbative selfconsistent way. Another effect we will consider are the vacuum polarization corrections arising from nucleons as already done in the QHD $[2,12,13]$. The equation of state for dense matter is derived. We organize the paper as follows: In Sec. II, we derive the equation of state (EOS) for dense matter including the quantum fluctuations from the nucleons and the $\sigma$ mesons within the QMC model. We also show the spectral function for the same level of approximation. In Sec. III, we discuss in detail the numerical results obtained in the present work and discuss possible outlook.

\section{THEORY}

The details of the QMC model have been given in Refs. $[3,4,6]$. Since we now include the vacuum polarization ef- 
fects of nucleons, we give here a few important steps for completeness.

In this model, the nucleon in nuclear matter is assumed to be described by a static MIT bag in which quarks interact with the scalar $(\sigma)$ and the vector $(\omega)$ mesons. The quark field $\psi_{q}(\mathbf{r}, t)$ inside the bag then satisfies the equation

$$
\left[i \gamma^{\mu} \partial_{\mu}-\left(m_{q}^{0}-g_{\sigma}^{q} \sigma_{0}\right)-g_{\omega}^{q} \omega_{0} \gamma^{0}\right] \psi_{q}(\mathbf{r}, t)=0,
$$

where $m_{q}^{0}$ is the current quark mass and $g_{\sigma}^{q}$ and $g_{\omega}^{q}$ are the quark couplings with the $\sigma$ and $\omega$ mesons.

After putting the boundary condition at the bag surface, we have the transcendental equation for the ground-state solution of the quark (in $s$ state) as

$$
j_{0}(x)=\beta_{q} j_{1}(x)
$$

which determines the bag eigenfrequency $x$. In the above, $\quad \beta_{q}=\sqrt{\left(\Omega_{q}-R m_{q}^{*}\right) /\left(\Omega_{q}+R m_{q}^{*}\right)}$, with $\quad \Omega_{q}=\left(x^{2}\right.$ $\left.+R^{2} m_{q}^{* 2}\right)^{1 / 2} ; m_{q}^{*}=m_{q}^{0}-g_{\sigma}^{q} \sigma_{0}$ is the effective quark mass. The form of the quark wave function is almost identical to that of the solution in free space. However, the parameters in the expression have been substantially modified by the surrounding nuclear medium. Thus the quarks in the nucleon embedded in the nuclear medium are more relativistic than those in a free nucleon.

The energy of the nucleon bag is

$$
M^{*}=3 \frac{\Omega_{q}}{R}-\frac{Z}{R}+\frac{4}{3} \pi R^{3} B
$$

where $B$ is the bag constant and $Z$ parametrizes the sum of the center-of-mass motion and the gluonic corrections. Note that this center-of-mass treatment is different from that of Jin and Jennings [10]. The bag radius $R$ is then obtained through $\partial M^{*} / \partial R=0$. This is the stability condition for the bag. An interesting fact related to the QMC model is that the bag volume changes in the medium through the mean value of the $\sigma$ field. This also implies that the bag eigenvalues are also modified.

\section{A. Vacuum polarization for nucleons}

We now proceed to study the EOS for nuclear matter including the vacuum polarization effects from nucleons at zero temperature. The details of the theory have already been discussed in the frame of QHD in Ref. [2] through an explicit construction of a state with nucleon-antinucleon condensates which is identical to those obtained through summing of tadpole diagrams for the baryon propagator in the relativistic Hartree approximation. Only a few important steps are given here. The energy density after subtracting out the pure vacuum contribution becomes

$$
\epsilon_{0}=\epsilon_{M F T}+\Delta \epsilon,
$$

with the mean field contribution given by

$$
\epsilon_{M F T}=\frac{\gamma}{(2 \pi)^{3}} \int_{|\mathbf{k}|<k_{F}} d \mathbf{k}\left(k^{2}+M^{* 2}\right)^{1 / 2}+\frac{1}{2} m_{\sigma}^{2} \sigma_{0}^{2}+\frac{1}{2} m_{\omega}^{2} \omega_{0}^{2},
$$

being the cutoff given by the (Fermi) momentum at the Fermi surface $k_{F}$, and

$$
\begin{aligned}
\Delta \epsilon= & -\frac{\gamma}{(2 \pi)^{3}} \int d \mathbf{k}\left[\left(k^{2}+M^{* 2}\right)^{1 / 2}\right. \\
& \left.-\left(k^{2}+M^{2}\right)^{1 / 2}-\frac{g_{\sigma} \sigma_{0} M}{\left(k^{2}+M^{2}\right)^{1 / 2}}\right] .
\end{aligned}
$$

The above expression for the energy density is divergent. After renormalization by adding the counterterms [12], we have the expression for the finite renormalized energy density,

$$
\epsilon_{r e n}=\epsilon_{M F T}+\Delta \epsilon_{r e n}
$$

where

$$
\begin{aligned}
\Delta \epsilon_{r e n}= & -\frac{\gamma}{16 \pi^{2}}\left[M^{* 4} \ln \left(\frac{M^{*}}{M}\right)+M^{3}\left(M-M^{*}\right)-\frac{7}{2} M^{2}(M\right. \\
& \left.\left.-M^{*}\right)^{2}+\frac{13}{3} M\left(M-M^{*}\right)^{3}-\frac{25}{12}\left(M-M^{*}\right)^{4}\right] .
\end{aligned}
$$

The baryonic density is obtained by

$$
\rho=\frac{\gamma k_{F}^{3}}{6 \pi^{2}}
$$

In the above, $\gamma$ is the spin-isospin degeneracy factor which is equal to 4 for nuclear matter and to 2 for neutron matter.

\section{B. Quantum fluctuations for the $\sigma$}

Next, we consider the quantum corrections due to the scalar meson. Including a quartic scalar self-interaction, the Hamiltonian density for the scalar mesons becomes

$$
\mathcal{H}_{\sigma}=\frac{1}{2} \partial_{\mu} \sigma \partial^{\mu} \sigma+\frac{1}{2} m_{\sigma}^{2} \sigma^{2}+\lambda \sigma^{4},
$$

with $m_{\sigma}$ and $\lambda$ being the bare mass and coupling constant, respectively. The quantized $\sigma$ field satisfies the algebra

$$
[\sigma(\mathbf{x}), \dot{\sigma}(\mathbf{y})]=i \delta(\mathbf{x}-\mathbf{y}) .
$$

We may expand the field operators in terms of creation and annihilation operators at time $t=0$ as

$$
\sigma(\mathbf{x})=\frac{1}{(2 \pi)^{3 / 2}} \int \frac{d \mathbf{k}}{\sqrt{2 \omega(\mathbf{k})}}\left[a(\mathbf{k})+a^{\dagger}(-\mathbf{k})\right] e^{i \mathbf{k} \cdot \mathbf{x}}
$$




$$
\dot{\sigma}(\mathbf{x})=\frac{i}{(2 \pi)^{3 / 2}} \int d \mathbf{k} \sqrt{\frac{\omega(\mathbf{k})}{2}}\left[-a(\mathbf{k})+a^{\dagger}(-\mathbf{k})\right] e^{i \mathbf{k} \cdot \mathbf{x}}
$$

In the above, $\omega(\mathbf{k})$ is an arbitrary function which for free fields is given by $\omega(\mathbf{k})=\sqrt{\mathbf{k}^{2}+m_{\sigma}^{2}}$ and the corresponding vacuum to this basis is defined through $a \mid$ vac $\rangle=0$. We shall now adopt a procedure as in Ref. [2] to calculate the quantum corrections arising from the $\sigma$ mesons. We consider an ansatz for the ground state including $\sigma$ condensates as

$$
|\Omega\rangle=U_{\sigma}|\mathrm{vac}\rangle,
$$

with

$$
U_{\sigma}=U_{I I} U_{I}
$$

where $U_{i}=\exp \left(B_{i}^{\dagger}-B_{i}\right)(i=I, I I)$. Explicitly the $B_{i}$ are given as

$$
B_{I}^{\dagger}=\int d \mathbf{k} \sqrt{\frac{\omega(\mathbf{k})}{2}} f_{\sigma}(\mathbf{k}) a^{\dagger}(\mathbf{k})
$$

and

$$
B_{I I}^{\dagger}=\frac{1}{2} \int d \mathbf{k} g(\mathbf{k}) a^{\prime \dagger}(\mathbf{k}) a^{\prime \dagger}(-\mathbf{k}) .
$$

In the above, $a^{\prime}(\mathbf{k})=U_{I} a(\mathbf{k}) U_{I}^{-1}=a(\mathbf{k})-\sqrt{\omega(\mathbf{k}) / 2} f_{\sigma}(\mathbf{k})$ corresponds to a shifted field operator associated with the coherent state [14] and satisfies the same algebra as the creation and annihilation operators for $\sigma$ and $\dot{\sigma}$. Thus in this construct for the ground state we have two functions $f_{\sigma}(\mathbf{k})$ and $g(\mathbf{k})$ which will be determined through minimization of energy density. Further, since $|\Omega\rangle$ contains an arbitrary number of $a^{\prime \dagger}$ quanta, $a^{\prime}|\Omega\rangle \neq 0$. However, we can define the basis $b(\mathbf{k}), b^{\dagger}(\mathbf{k})$ corresponding to $|\Omega\rangle$ through the Bogoliubov transformation. Further, to preserve translational invariance, $f_{\sigma}(\mathbf{k})$ has to be proportional to $\delta(\mathbf{k})$ and we take $f_{\sigma}(\mathbf{k})=\sigma_{0}(2 \pi)^{3 / 2} \delta(\mathbf{k}) . \quad \sigma_{0}$ will correspond to a classical field of the conventional approach [14]. It is easy to evaluate that

$$
\langle\Omega|\sigma| \Omega\rangle=\sigma_{0},
$$

but,

$$
\left\langle\Omega\left|\sigma^{2}\right| \Omega\right\rangle=\sigma_{0}^{2}+I,
$$

where

$$
I=\frac{1}{(2 \pi)^{3}} \int \frac{d \mathbf{k}}{2 \omega(k)}(\cosh 2 g+\sinh 2 g) .
$$

We next calculate the expectation value of the Hamiltonian density for the $\sigma$ meson given by Eq. (10). Using Eqs. (18)(20) the energy density of $\mathcal{H}_{\sigma}$ with respect to the trial state becomes

$$
\begin{aligned}
\epsilon_{\sigma} \equiv & \left\langle\Omega\left|\mathcal{H}_{\sigma}\right| \Omega\right\rangle=\frac{1}{2} \frac{1}{(2 \pi)^{3}} \int \frac{d \mathbf{k}}{2 \omega(k)}\left[k^{2}(\sinh 2 g+\cosh 2 g)\right. \\
& \left.+\omega^{2}(k)(\cosh 2 g-\sinh 2 g)\right]+\frac{1}{2} m_{\sigma}^{2} I+6 \lambda \sigma_{0}^{2} I+3 \lambda I^{2} \\
& +\frac{1}{2} m_{\sigma}^{2} \sigma_{0}^{2}+\lambda \sigma_{0}^{4} .
\end{aligned}
$$

Extremizing the above energy density with respect to the function $g(k)$ yields

$$
\tanh 2 g(k)=-\frac{6 \lambda I+6 \lambda \sigma_{0}^{2}}{\omega(k)^{2}+6 \lambda I+6 \lambda \sigma_{0}^{2}} .
$$

It is clear from the above equation that in the absence of a quartic coupling no such averaged value is favored since this function vanishes for $\lambda=0$. Now substituting this value of $g(k)$ in the expression for the $\sigma$-meson energy density yields

$$
\boldsymbol{\epsilon}_{\sigma}=\frac{1}{2} m_{\sigma}^{2} \sigma_{0}^{2}+\lambda \sigma_{0}^{4}+\frac{1}{2} \frac{1}{(2 \pi)^{3}} \int d \mathbf{k}\left(k^{2}+M_{\sigma}^{2}\right)^{1 / 2}-3 \lambda I^{2},
$$

where

$$
M_{\sigma}^{2}=m_{\sigma}^{2}+12 \lambda I+12 \lambda \sigma_{0}^{2}
$$

with

$$
I=\frac{1}{(2 \pi)^{3}} \int \frac{d \mathbf{k}}{2} \frac{1}{\left(\mathbf{k}^{2}+M_{\sigma}^{2}\right)^{1 / 2}}
$$

obtained from Eq. (20) after substituting for the condensate function $g(k)$ as in Eq. (22). This expression (25) defines the $\sigma$ mass $M_{\sigma}$. In Eq. (23) for the "effective potential" $\epsilon_{\sigma}$ contains divergent integrals. However, our approximation is nonperturbatively self-consistent and it still contains the infinities in the integral $I$ given by Eq. (25) which need to be eliminated also in order to reinforce the meaning of $M_{\sigma}$. Therefore we first obtain a well-defined finite expression for $M_{\sigma}$ by renormalization. We use the regularization by cutoff and the renormalization prescription of Ref. [15] and thus obtain the renormalized mass $m_{R}$ and coupling $\lambda_{R}$ through

$$
\begin{aligned}
& \frac{m_{R}^{2}}{\lambda_{R}}=\frac{m^{2}}{\lambda}+12 I_{1}(\Lambda), \\
& \frac{1}{\lambda_{R}}=\frac{1}{\lambda}+12 I_{2}(\Lambda, \mu),
\end{aligned}
$$

where $I_{1}$ and $I_{2}$ are the integrals,

$$
\begin{gathered}
I_{1}(\Lambda)=\frac{1}{(2 \pi)^{3}} \int_{|\mathbf{k}|<\Lambda} \frac{d \mathbf{k}}{2 k}, \\
I_{2}(\Lambda)=\frac{1}{\mu^{2}(2 \pi)^{3}} \int_{|\mathbf{k}|<\Lambda} d \mathbf{k}\left(\frac{1}{2 k}-\frac{1}{2 \sqrt{k^{2}+\mu^{2}}}\right),
\end{gathered}
$$


TABLE I. Parameters used in the calculation.

\begin{tabular}{lcccccc}
\hline \hline$M(\mathrm{MeV})$ & $m_{q}(\mathrm{MeV})$ & $R(\mathrm{fm})$ & $B^{1 / 4}(\mathrm{MeV})$ & $Z$ & $m_{R}(\mathrm{MeV})$ & $m_{\omega}(\mathrm{MeV})$ \\
\hline 939.0 & 0.0 & 0.6 & 211.303 & 3.9869 & 550.0 & 783.0 \\
\hline \hline
\end{tabular}

where $\mu$ is a renormalization mass scale and $\Lambda$ is the ultraviolet cutoff. The resulting gap equation for $M_{\sigma}^{2}$, which minimizes the energy, in terms of the renormalized parameters $m_{R}^{2}$ and $\lambda_{R}$ can be rewritten as

$$
M_{\sigma}^{2}=m_{R}^{2}+12 \lambda_{R} \sigma_{0}^{2}+12 \lambda_{R} I_{f}\left(M_{\sigma}\right),
$$

where $\sigma_{0}$ is obtained from the minimization of the energy density with relation to $\sigma$,

$$
\left.\frac{d \epsilon}{d \sigma}\right|_{\sigma_{0}}=0,
$$

and

$$
I_{f}\left(M_{\sigma}\right)=\frac{M_{\sigma}^{2}}{16 \pi^{2}} \ln \left(\frac{M_{\sigma}^{2}}{\mu^{2}}\right) .
$$

Self-consistency in expression (30) makes the $\sigma$ mass to depend on the nucleon polarization because $\sigma_{0}$ does depend on it. Using the above equations we obtain the energy density for the $\sigma$ in terms of $\sigma_{0}$ which is given by

$$
\begin{aligned}
\epsilon_{\sigma}= & 3 \lambda_{R}\left(\sigma_{0}^{2}+\frac{m_{R}^{2}}{12 \lambda_{R}}\right)^{2}+\frac{M_{\sigma}^{4}}{64 \pi^{2}}\left[\ln \left(\frac{M_{\sigma}^{2}}{\mu^{2}}\right)-\frac{1}{2}\right] \\
& -3 \lambda_{R} I_{f}^{2}-2 \lambda \sigma_{0}^{4},
\end{aligned}
$$

where $\mu$ is a mass scale introduced in the renormalization procedure. The above expression is given in terms of $\sigma$ mass, $m_{R}$, and $\lambda_{R}$. However, from the renormalization procedure one sees that when $\lambda_{R}$ is kept fixed, the bare coupling $\lambda \rightarrow 0{ }_{-}$. Therefore the last term in Eq. (33) will be neglected in the numerical calculations. This approximation is nearly equivalent to taking into account the so-called "cactus" diagrams [16] which correspond to a resummation of the tadpole diagram with the loop propagator given by the selfenergy self-consistently.

After subtracting the vacuum contribution we obtain

$$
\begin{aligned}
\Delta \epsilon_{\sigma}= & \epsilon_{\sigma}-\epsilon_{\sigma}\left(\sigma_{0}=0\right) \\
= & \frac{1}{2} m_{R}^{2} \sigma_{0}^{2}+3 \lambda_{R} \sigma_{0}^{4}+\frac{M_{\sigma}^{4}}{64 \pi^{2}}\left[\ln \left(\frac{M_{\sigma}^{2}}{\mu^{2}}\right)-\frac{1}{2}\right] \\
& -3 \lambda_{R} I_{f}^{2}-\frac{M_{\sigma, 0}^{4}}{64 \pi^{2}}\left[\ln \left(\frac{M_{\sigma, 0}^{2}}{\mu^{2}}\right)-\frac{1}{2}\right]+3 \lambda_{R} I_{f 0}^{2},
\end{aligned}
$$

where $M_{\sigma, 0}$ and $I_{f 0}$ are the expressions given by Eqs. (30) and (32) with $\sigma_{0}=0$.

The energy density and pressure with baryon and the sigma condensate $\sigma_{0}$ are, respectively, done by

$$
\epsilon_{\text {ren }}=\epsilon_{0}^{\text {finite }}+\Delta \epsilon_{\text {ren }},
$$

and

$$
\begin{aligned}
P= & \frac{\gamma}{3(2 \pi)^{3}} \int_{|\mathbf{k}|<k_{F}} d \mathbf{k} \frac{k^{2}}{\left(k^{2}+M^{* 2}\right)^{1 / 2}}+\frac{1}{2} m_{\omega}^{2} \omega_{0}^{2} \\
& -\Delta \boldsymbol{\epsilon}_{\sigma}-\Delta \boldsymbol{\epsilon}_{r e n},
\end{aligned}
$$

where

$$
\epsilon_{0}^{\text {finite }}=\frac{\gamma}{(2 \pi)^{3}} \int_{|\mathbf{k}|<k_{F}} d \mathbf{k}\left(k^{2}+M^{* 2}\right)^{1 / 2}+\frac{1}{2} m_{\omega}^{2} \omega_{0}^{2}+\Delta \epsilon_{\sigma}
$$

with $\Delta \epsilon_{r e n}$ given by Eq. (8) and $\Delta \epsilon_{\sigma}$ by Eq. (34). In the above equation, the last term includes the contribution from the quantum correction of the $\sigma$ mesons. In the mean field approximation, i.e., in normal QMC model, $\lambda_{R}=0$, and the energy density from the $\sigma$ meson is $\frac{1}{2} m_{R}^{2} \sigma_{0}^{2}$. This then corresponds to the relativistic Hartree approximation (RHA), with the last term of Eq. (35) being the contribution arising from the vacuum polarization effects from nucleon sector. The usual vector mean field $\omega_{0}$ is obtained from its equation of motion as

$$
\omega_{0}=\frac{3 g_{\omega}^{q}}{m_{\omega}^{2}} \rho .
$$

The energy density from the $\sigma$ field as given by Eq. (34) is still in terms of the renormalization scale $\mu$ which is arbitrary. We choose this to be equal to the renormalized $\sigma$ mass $m_{R}$ in doing the numerical calculations. This is because changing $\mu$ would mean changing the quartic coupling $\lambda_{R}$, and this coupling constant enters here as a parameter to be chosen to give the incompressibility for nuclear matter in the correct range. The parameters $g_{\sigma}^{q}$ and $g_{\omega}\left(=3 g_{\omega}^{q}\right)$ are fitted so as to describe the ground-state properties of nuclear matter correctly. For a given baryon density $\rho$, the energy density, the density dependent radius of the nucleon and the nucleon effective mass are calculated at zero temperature.

\section{RESULTS AND DISCUSSION}

We now proceed with the numerical calculations for the nuclear matter taking into account both effects presented above. We start fixing the bag properties in the vacuum. We use zero quark masses and the bag radius $R=0.6 \mathrm{fm}$. There are two unknowns, $Z$ and the bag constant $B$. These are obtained as usual by fitting the nucleon mass $M=939 \mathrm{MeV}$ and enforcing the stability condition for the bag. They are given in Table I. 


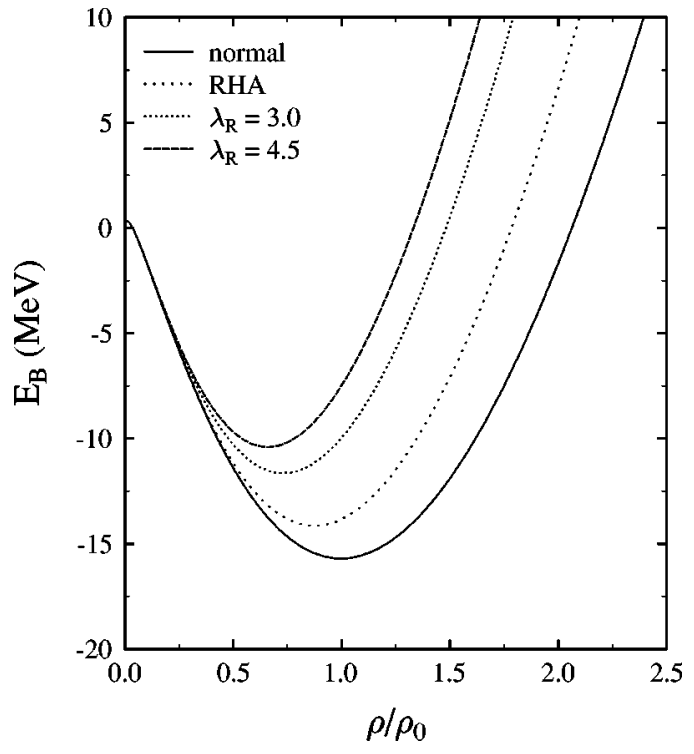

FIG. 1. The energy per nucleon of nuclear matter as a function of $\rho / \rho_{0}$ for different corrections. All curves are for the same set of parameter of QMC.

We next calculate the ground-state properties of the nuclear matter and fit the scalar and vector coupling constants $g_{\sigma}^{q}$ and $g_{\omega}\left(=3 g_{\omega}^{q}\right)$ to reproduce the nuclear matter binding energy $(-15.7 \mathrm{MeV})$ at the saturation density $\left(\rho_{0}\right.$ $=0.15 \mathrm{fm}^{-3}$ ) for a given renormalized $\sigma$ mass and coupling, $m_{R}$ and $\lambda_{R}$.

Initially, we investigate the effect of the quantum corrections on the binding energy per particle arising from the nucleon and the $\sigma$ mesons. The results are shown in Fig. 1, where we plot $E_{B}$ as a function of the nuclear density $\rho / \rho_{0}$. In this figure the coupling constants $g_{\sigma}^{q}$ and $g_{\omega}$ are the same for the RHA, $\lambda_{R}=3.0$, and $\lambda_{R}=4.5$ cases. Observe that the saturation density shifts by $\simeq 0.15 \rho_{0}$ for RHA and $\simeq 0.5 \rho_{0}$ for $\lambda_{R}=3.0$ and the binding energy changes $\simeq 1.5 \mathrm{MeV}$ for RHA and $\simeq 4 \mathrm{MeV}$ for $\lambda_{R}=3.0$ when the fluctuations are included. In this way it is possible to assess the quantitative relevance of including quantum fluctuations for the $\sigma$. However the parameters must be readjusted in order to describe ground-state nuclear matter properties.

We now readjust the coupling constants $g_{\sigma}^{q}$ and $g_{\omega}$ such as to obtain the correct saturation binding energy of nuclear matter for RHA and for different $\lambda_{R}$. The $\omega$ and $\sigma$ couplings for given $\lambda_{R}$ are tabulated in Table II. Results from RHA are

TABLE II. Quark- $\sigma$ and $\omega$-nucleon coupling constants, inmedium nucleon properties at saturation density, and the nuclear matter incompressibility for different cases.

\begin{tabular}{lccccc}
\hline \hline Case & $g_{s}^{q}$ & $g_{\omega}$ & $M^{*} / M_{N}$ & $R^{*}(\mathrm{fm})$ & $K(\mathrm{MeV})$ \\
\hline Normal QMC & 5.98 & 8.96 & 0.775 & 0.5961 & 281 \\
RHA & 5.77 & 8.39 & 0.793 & 0.5967 & 272 \\
$\lambda_{R}=2.0$ & 5.60 & 8.02 & 0.809 & 0.5972 & 263 \\
$\lambda_{R}=3.0$ & 5.51 & 7.77 & 0.817 & 0.5975 & 256 \\
$\lambda_{R}=4.5$ & 5.37 & 7.36 & 0.830 & 0.5978 & 244 \\
\hline \hline
\end{tabular}

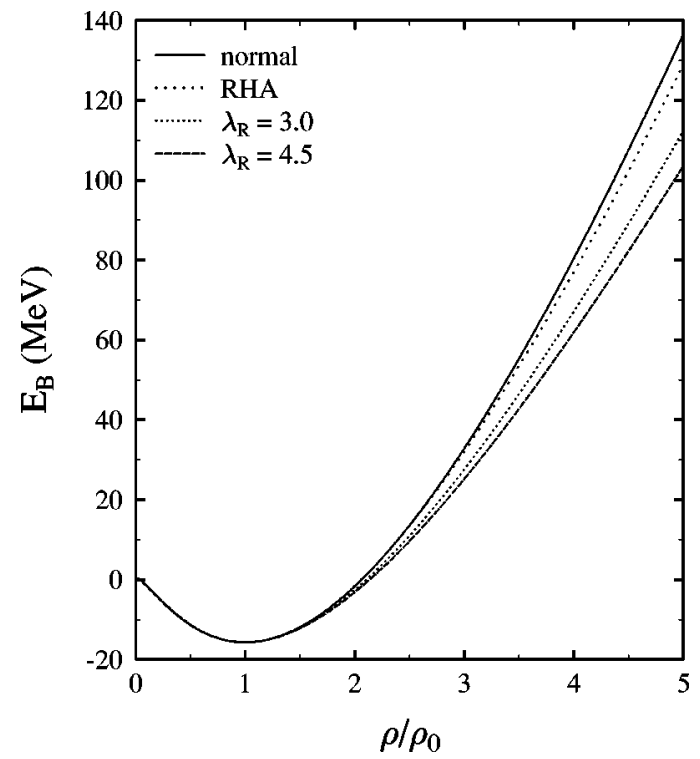

FIG. 2. The binding energy of the nuclear matter as a function of $\rho / \rho_{0}$. The parameters are refitted to the saturation properties of nuclear matter at the minimum of the curves.

also shown. Little change is noted in the values of the parameters for the range of $\lambda_{R}$ which is considered here. The value of $\sigma_{0}$ which enters, for example, in the $\sigma$ mass, is determined by the minimization of expression (35) with respect to $\sigma$ at each density in a self-consistent manner. Using these values, we plot the binding energy $\left(E_{B}=\epsilon / \rho-M_{N}\right)$ for nuclear matter as a function of density in Fig. 2. In the same figure we also plot the results for the RHA. Clearly, including baryon and $\sigma$-meson quantum corrections leads to a softer equation of state which is further softer for a higher value of $\lambda_{R}$. The equation of state, pressure, $P$ versus as a function of energy density $\epsilon$ is displayed in Fig. 3 for different cases. For comparison, the causal limit $P=\epsilon$ is also shown in the figure. All the cases studied here respect the

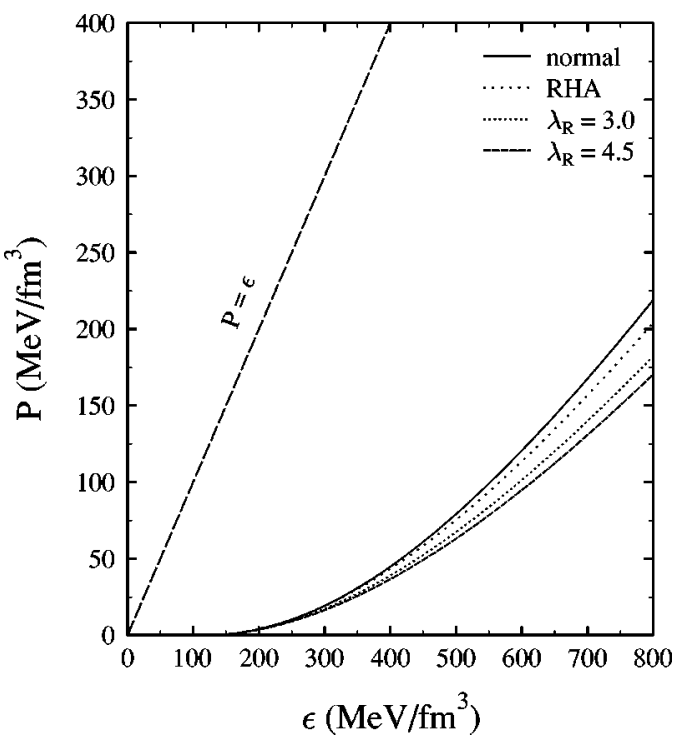

FIG. 3. The pressure versus energy density of the nuclear matter. 


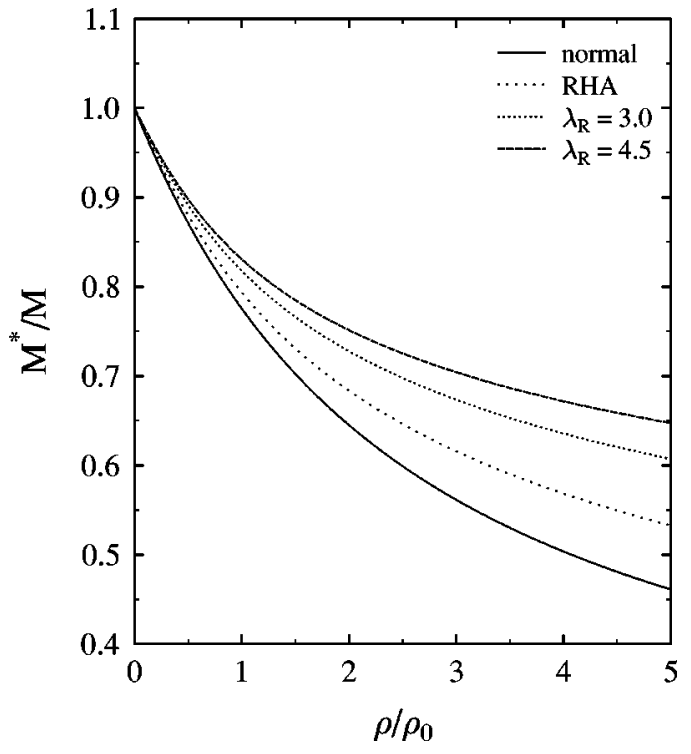

FIG. 4. Effective baryon masses in the medium.

causal condition $\partial P / \partial \epsilon \leqslant 1$, so that the speed of sound remains lower than the speed of light.

In Fig. 4 we plot the effective nucleon mass as a function of density. At the saturation density we get $M^{*}=0.817 M$ and $0.83 M$ for $\lambda_{R}=3.0$ and $\lambda_{R}=4.5$, respectively. These values may be compared with the results of $M^{*}=0.775 M$ for normal QMC model and of $0.793 M$ with the relativistic Hartree approximation in the QMC model keeping the coupling constants fixed. This influence is much higher at high nuclear densities. We can conclude that quantum effects, at the level we consider, increase the effective mass $M^{*}$.

We plot the in-medium effective radius of the nucleon $\left(R^{*}\right)$ as a function of density in Fig. 5. $R^{*}$ is also increased in relation to the mean field approximation mainly for higher densities.

Next, the spectral function for the $\sigma$ mesons including the

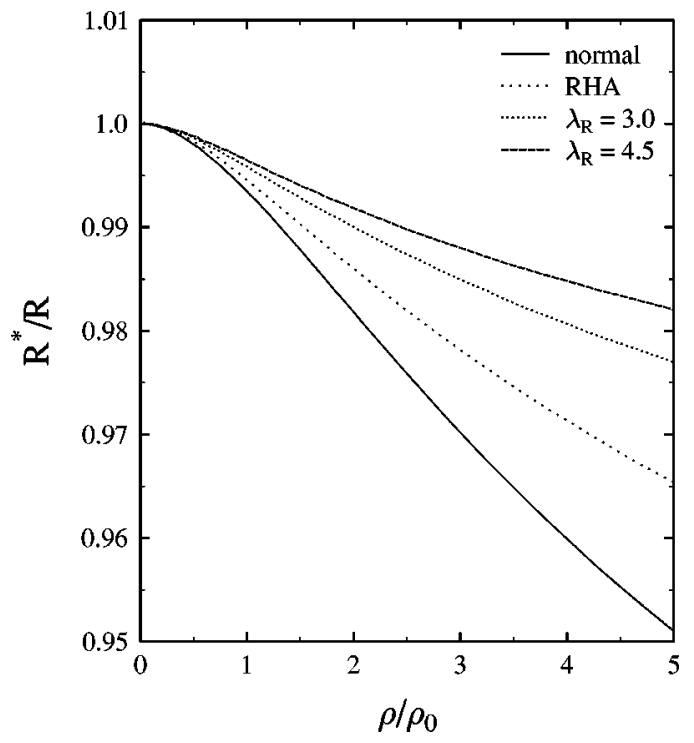

FIG. 5. The effective radius of the nucleon as a function of $\rho / \rho_{0}$.

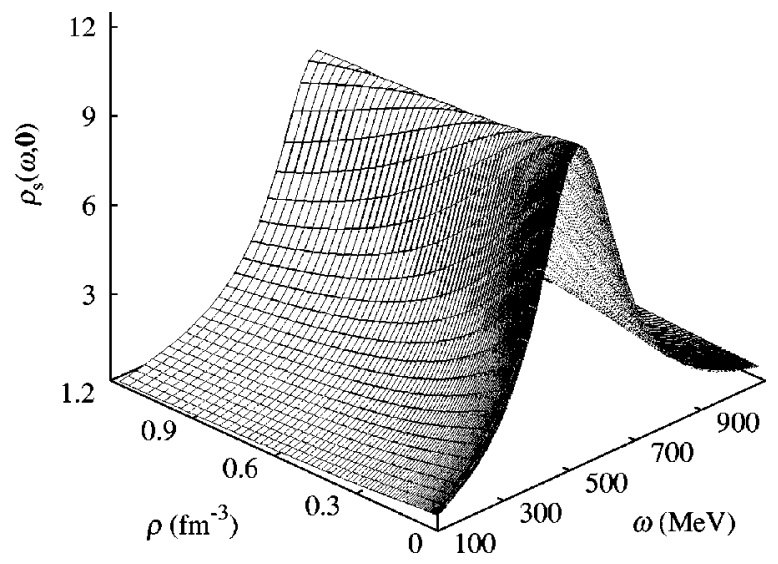

FIG. 6. The spectral function (in units of $M^{-2}$ ) of $\sigma$ meson for $\lambda_{R}=3.0$.

quantum correction effects is examined [17]. It is very interesting to study these functions because, for example, in a thermal model the dilepton yields in heavy-ion collisions would be proportional to the spectral function. It gives a more complete account of the in-medium $\sigma$ properties as mass and width.

The spectral function in the quantum theory can be expressed in terms of the retarded self-energy, $\Sigma_{R}=\operatorname{Re} \Sigma_{R}$ $+i \operatorname{Im} \Sigma_{R}$, as

$\rho_{s}(\omega, \mathbf{p})$

$$
=\frac{-2 \operatorname{Im} \Sigma_{R}(\omega, \mathbf{p})}{\left[\omega^{2}-\mathbf{p}^{2}-m_{R}^{2}-\operatorname{Re} \Sigma_{R}(\omega, \mathbf{p})\right]^{2}+\left[\operatorname{Im} \Sigma_{R}(\omega, \mathbf{p})\right]^{2}} .
$$

By definition the width of the corresponding mode, the $\sigma$, is given by $\Gamma=-\operatorname{Im} \Sigma_{R}(\omega, \mathbf{p}) / \omega$. While the real part of the self-energy is directly related to the (density dependent) $\sigma$ mass, which was calculated in the preceding section, the imaginary part will be defined by a width of the $\sigma$ considered to be constant at this level of approximation. It will be given by $\Gamma(\rho)=300 \mathrm{MeV}$ [18]. This is an ad hoc prescription that does not prevent the imaginary part of the spectral function from varying with the density. The spectral function at $\mathbf{p}$ $=0$ can then be written in terms of it as

$$
\rho_{s}(\omega, \mathbf{0})=\frac{2 \omega \Gamma}{\left(\omega^{2}-M_{\sigma}^{2}\right)^{2}+\omega^{2} \Gamma^{2}},
$$

where $M_{\sigma}^{2}$ is given by expression (30).

In Fig. 6, we plot the spectral function of the $\sigma$ meson as a function of the energy and of the density. The spectral function becomes more strengthened as density increases and its center moves slightly for higher energies. We found therefore that $M_{\sigma}$ increases with density as $\lambda_{R}$ is positive, although this is not so clear in the graph. The resulting width of the spectral function is smaller at higher densities. In Fig. 7 , it is explicitly shown the $\sigma$ mass as a function of the ratio 


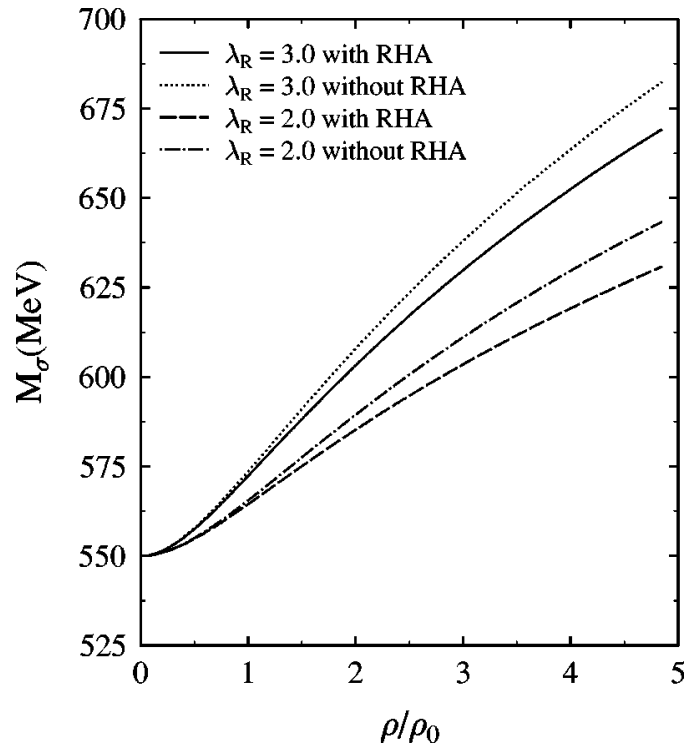

FIG. 7. The $\sigma$ self-consistent mass for $\lambda_{R}=3.0$ and $\lambda_{R}=2.0$ with and without considering the polarization effects for nucleons.

$\rho / \rho_{0}$ for two couplings $\lambda_{R}=2.0$ and $\lambda_{R}=3.0$ with and without the nucleon polarization effects. We note that while the nucleon polarization effects (RHA for a fixed coupling $\lambda_{R}$ ) tend to make the $\sigma$ mass smaller, the quantum fluctuations of the $\sigma$ make its mass to increase. This last effect is dominant at finite densities even for couplings as weak as $\lambda_{R}=2.0$.
The difference of the $\sigma$ mass due to the nucleon polarization is nearly independent of $\lambda_{R}$. The higher is the $(\sigma)$ coupling $\lambda_{R}$ the higher is the increase of the $\sigma$ mass with density. This seems to be rather an indication of a further symmetry breaking instead of its restoration [11].

To summarize, we have used a nonperturbative approach to include the quantum effects in nuclear matter in the framework of QMC. The calculations of the scalar meson quantum corrections was done here in a self-consistent manner including multiloop effects. This leads to a softening of the equation of state. We have also calculated the spectral function of the $\sigma$ and, in particular, the effective in-medium mass of the $\sigma$ meson modified by the quantum corrections. The effective $\sigma$ mass increases with density although the polarization due to nucleons contributes to diminish this mass. This may be understood as a tendency to a prevention from (chiral) symmetry restoration. These features deserve further investigation as well as the role of the coupling constant $\lambda_{R}$ and its influence on the chiral symmetry behavior at high densities and temperatures.

\section{ACKNOWLEDGMENTS}

The authors would like to thank G. Krein for extensive discussions and many useful suggestions. P.K.P. would like to acknowledge FAPESP (Grant No. 99/08544-0) for financial support and the IFT, São Paulo, for kind hospitality. F.L.B. was supported by FAPESP (Grant No. 97/01317-3).
[1] B.D. Serot and J.D. Walecka, Adv. Nucl. Phys. 16, 1 (1986); Int. J. Mod. Phys. E 6, 515 (1997); J.D. Walecka, Ann. Phys. (N.Y.) 83, 491 (1974).

[2] A. Mishra, P.K. Panda, S. Schramm, J. Reinhardt, and W. Greiner, Phys. Rev. C 56, 1380 (1997); P.K. Panda, R. Sahu, and S. Mishra, ibid. 62, 055802 (2000); A. Mishra, P.K. Panda, and W. Greiner, J. Phys. G 27, 1561 (2001); 28, 67 (2002).

[3] P.A.M. Guichon, Phys. Lett. B 200, 235 (1988).

[4] K. Saito and A.W. Thomas, Phys. Lett. B 327, 9 (1994); 335, 17 (1994); 363, 157 (1995); Phys. Rev. C 52, 2789 (1995); P.A.M. Guichon, K. Saito, E. Rodionov, and A.W. Thomas, Nucl. Phys. A601, 349 (1996); K. Saito, K. Tsushima, and A.W. Thomas, ibid. A609, 339 (1996); Phys. Rev. C 55, 2637 (1997); Phys. Lett. B 406, 287 (1997); D.H. Lu, K. Tsushima, A.W. Thomas, A.G. Williams, and K. Saito, Nucl. Phys. A634, 443 (1998).

[5] P.G. Blunden and G.A. Miller, Phys. Rev. C 54, 359 (1996); G. Krein, D.P. Menezes, M. Nielsen, and C. Providencia, Nucl. Phys. A674, 125 (2000); N. Barnea and T.S. Walhout, ibid. A677, 367 (2000); H. Shen and H. Toki, Phys. Rev. C 61, 045205 (2000).

[6] P.K. Panda, A. Mishra, J.M. Eisenberg, and W. Greiner, Phys. Rev. C 56, 3134 (1997).
[7] P.K. Panda, R. Sahu, and C. Das, Phys. Rev. C 60, 038801 (1999).

[8] G. Krein, A.W. Thomas, and K. Tsushima, Nucl. Phys. A650, 313 (1999); M.E. Bracco, G. Krein, and M. Nielsen, Phys. Lett. B 432, 258 (1998).

[9] P.K. Panda, M.E. Bracco, M. Chiapparini, E. Conte, and G. Krein, Phys. Rev. C 65, 065206 (2002).

[10] X. Jin and B.K. Jennings, Phys. Rev. C 54, 1427 (1996); Phys. Lett. B 374, 13 (1996).

[11] F.L. Braghin, Phys. Rev. D 64, 125001 (2001).

[12] S.A. Chin and J.D. Walecka, Phys. Lett. B 52, 24 (1974); S.A. Chin, Ann. Phys. (N.Y.) 108, 301 (1977); R.J. Perry, Phys. Lett. B 199, 489 (1987).

[13] N.K. Glendenning, Nucl. Phys. A493, 521 (1989).

[14] A. Mishra and H. Mishra, J. Phys. G 23, 143 (1997).

[15] S. Coleman, R. Jackiw, and H.D. Politzer, Phys. Rev. D 10, 2491 (1974).

[16] T. Barnes and G.I. Ghandhour, Phys. Rev. D 22, 924 (1980).

[17] K. Saito, K. Tsushima, A.W. Thomas, and A.G. Williams, Phys. Lett. B 433, 243 (1998); K. Saito, K. Tsushima, and A.W. Thomas, ibid. 460, 17 (1999).

[18] N.A. Törnqvist and M. Roos, Phys. Rev. Lett. 76, 1575 (1996); M. Harada, F. Sannino, and J. Schechter, Phys. Rev. D 54, 1991 (1996); Phys. Rev. Lett. 78, 1603 (1997); S. Ishida et al., Prog. Theor. Phys. 98, 1005 (1997). 